\begin{tabular}{|c|c|c|c|}
\hline \multicolumn{4}{|c|}{ COMPARISON OF ASTRONOMICAL AND DEVILS HOLE ICE AGES } \\
\hline \multirow[b]{2}{*}{ Bathythermal } & \multicolumn{2}{|c|}{ Chronology (years before present) } & \multirow{2}{*}{$\begin{array}{l}\text { Cycle duration } \\
\text { (years) }\end{array}$} \\
\hline & Devils Hole & Astronomical & \\
\hline 2 & - & 25,000 & \\
\hline 4 & 70,000 & 70,000 & \\
\hline 6 & 160,000 & 150,000 & 80,000 \\
\hline & & & 120,000 \\
\hline 8 & 270,000 & 270,000 & \\
\hline 10 & 358,000 & 355,000 & \\
\hline 12 & 450,000 & 435,000 & 80,000 \\
\hline & & & 120,000 \\
\hline 14 & 550,000 & 555,000 & \\
\hline
\end{tabular}

in duration one, two or three obliquity cycles, an apparent result of beating between the obliquity and eccentricity cycles 8 . Before about one million years ago, such beating was much more sporadic and obliquity was the dominant factor timing the ice ages $^{7}$. Although it has long been recognized that eccentricity has a secondary effect on climate compared to obliquity, eccentricity may become important at

period) to an average of about 90,000 years, and the amplitude increased, with the bathythermals deepening to produce intense glaciations ${ }^{7}$.

The last column in the table shows that the 'periodicity' of 90,000 years actually derives from the isotope cycles ranging times when other conditions (the Tibetan uplift, for instance ${ }^{9}$ ) have driven climate to criticality.

\section{Cesare Emiliani}

Department of Geological Sciences,

University of Miami,

Coral Gables, Florida 33124, USA

\title{
ALS, SOD and peroxynitrite
}

SIR - Amyotrophic lateral sclerosis (ALS) is characterized by degeneration of large motor neuron nuclei in the spinal cord. Because muscle fibres are innervated by only a single motor neuron, each loss is particularly destructive. Interneurons surrounding motor nuclei produce nitric oxide that helps modulate synaptic plasticity ${ }^{1}$ and may indirectly contribute to motor neuron destruction in ALS by $\mathrm{Cu}, \mathrm{Zn}$ superoxide dismutase (SOD). Eleven unique mutations at nine distinct sites in SOD are associated with the autosomal dominant inheritance of familial ALS (see figure) ${ }^{2}$. Because afflicted ALS patients have one normal

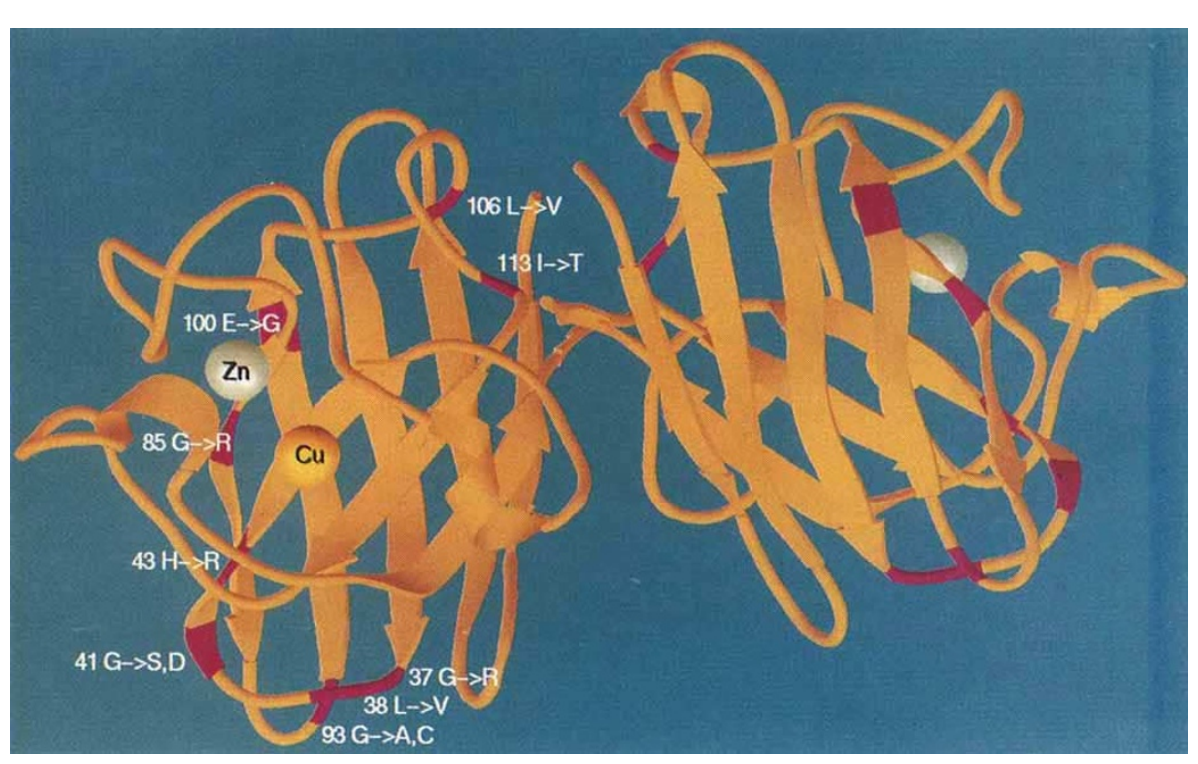

Protein backbone of human $\mathrm{Cu}, \mathrm{Zn} \mathrm{SOD}$ is shown looking down the active site to the copper, based on the human X-ray structure ${ }^{8}$. Mutant amino-acid substitutions are shown in red and are primarily localized on the ends of the $\beta$-barrel. Mutations at these diverse sites may push open the loops that form the superoxide-binding pocket and expose more of the copper for reaction with peroxynitrite.
The mutations identified in ALS patients do not directly affect conserved amino acids forming the active site, but could slightly disrupt the active-site pocket to allow greater access of peroxynitrite to the copper (see figure). Chemical modification of several amino acids in the active site allows SOD to react with peroxynitrite but greatly diminishes superoxide scavenging ${ }^{4}$. Thus, the SOD mutations may increase both peroxynitrite formation owing to reduced scavenging of superoxide and nitration by peroxynitrite of critical cellular targets.

Nitration of proteins will slowly injure motor neurons as well as other cells. However, motor neurons cannot regenerate. Motor neuron injury will accelerate as the nervous system adapts by rearranging synapses to other motor neurons, subjecting them to additional fluxes of nitric oxide. Peripheral nerve injury and chronic inflammation can induce nitric oxide synthase in spinal cord ${ }^{5,6}$. Motor neurons may also possess a target that is unusually susceptible to nitration, such as tyrosine kinases. For example, brain-derived neurotrophic factor rescues spinal motor neurons from cell death in vivo and binds to the receptor tyrosine kinase trkB (ref. 7). Altered phosphorylation also affects the abnormal assembly and distribution of neurofilaments observed in ALS.

Because native SOD also catalyses nitration by peroxynitrite, ALS in some patients with normal SOD genes may result from peroxynitrite formation due to mild trauma or inflammation near motor neurons. Therefore, caution should be exercised before attempting to treat ALS patients with SOD until the mechanisms accounting for the dominant action of the SOD mutants are better understood.

\section{Joseph S. Beckman}

Department of Anesthesiology,

Michael Carson

Craig D. Smith

Center for Macromolecular

Crystallography,

University of Alabama at Birmingham,

Birmingham,

Alabama 35233, USA

Willem H. Koppenol

Department of Chemistry,

Louisiana State University,

Baton Rouge,

Louisiana 70803, USA

1. Vaitschanoff, J. G., Weinberg, R. J. \& Rustioni, A. J. comp. Neurol. 321, 209-222 (1992)

2. Rosen, D. R. etal. Nature 362, 59-62 (1993).

3. Huie, R. E. \& Padmaja, S. Free Rad. Res. Commun. 18, 195-201(1993).

4. Beckman, J.S. etal. Archs biochem. Biophys. 298, 438-445 (1992).

5. Verge, V. M. K., Xu, Z., Xu, X. J., Wiesenfeld-Hallin, Z. \& Hökfelt, T. Proc. natn. Acad. Sci. U.S.A. 89, 1161711621 (1992).

6. Solodkin, A., Traub, R. J. \& Gebhart, G. F. Neuroscience 51, 495-499(1992).

7. Oppenheim, R. W., Qin-Wei, Y., Prevett, D. \& Yan, Q Nature 360, 755 (1992).

8. Parge, H. E., Hallewell, R. A. \& Tainer, J. A. Proc. natn. Acad. Sci.U.S.A. 89, 6109-6113(1992). 